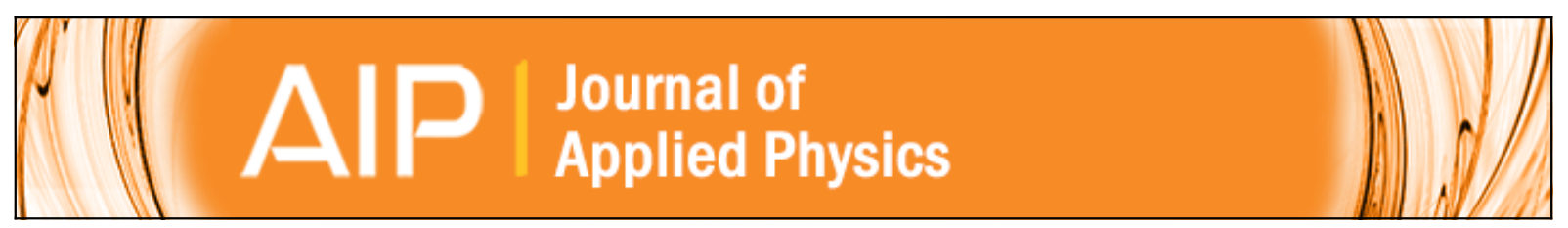

\title{
Irreversible entropy model for damage diagnosis in resistors
}

Angel Cuadras, Javier Crisóstomo, Victoria J. Ovejas, and Marcos Quilez

Citation: Journal of Applied Physics 118, 165103 (2015); doi: 10.1063/1.4934740

View online: http://dx.doi.org/10.1063/1.4934740

View Table of Contents: http://scitation.aip.org/content/aip/journal/jap/118/16?ver=pdfcov

Published by the AIP Publishing

\section{Articles you may be interested in}

The Sponge Resistor Model - A Hydrodynamic Analog to Illustrate Ohm's Law, the Resistor Equation R $=\rho \ell / \mathrm{A}$ , and Resistors in Series and Parallel

Phys. Teach. 52, 270 (2014); 10.1119/1.4872404

Resistors Network Model of Bcc Cell for Investigating Thermal Conductivity of Nanofluids

AIP Conf. Proc. 1415, 86 (2011); 10.1063/1.3667227

Entropy and irreversibility in the quantum realm Am. J. Phys. 79, 297 (2011); 10.1119/1.3533719

A three-dimensional resistor network model for the linear magnetoresistance of $\mathrm{Ag} 2+\delta$ Se and $\mathrm{Ag} 2+\delta \mathrm{Te}$ bulks

J. Appl. Phys. 104, 113922 (2008); 10.1063/1.3035834

Irreversibility, Time Reversal and Generalised Entropy

AIP Conf. Proc. 734, 383 (2004); 10.1063/1.1834458

\section{AIP $\mid$ APL Photonics}

APL Photonics is pleased to announce Benjamin Eggleton as its Editor-in-Chief

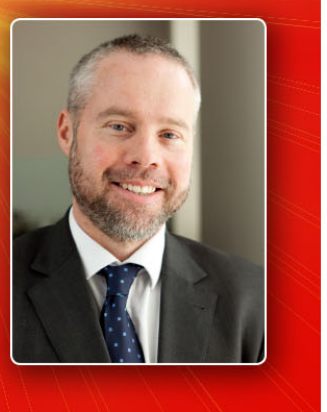




\title{
Irreversible entropy model for damage diagnosis in resistors
}

\author{
Angel Cuadras, ${ }^{\text {a) }}$ Javier Crisóstomo, Victoria J. Ovejas, and Marcos Quilez \\ Instrumentation, Sensor and Interfaces Group, Electronic Engineering Department, Escola d' Enginyeria de \\ Telecomunicació i Aeronàutica de Castelldefels EETAC, Universitat Politècnica de Catalunya, Barcelona \\ Tech (UPC), Castelldefels-Barcelona, Spain
}

(Received 29 July 2015; accepted 15 October 2015; published online 29 October 2015)

\begin{abstract}
We propose a method to characterize electrical resistor damage based on entropy measurements. Irreversible entropy and the rate at which it is generated are more convenient parameters than resistance for describing damage because they are essentially positive in virtue of the second law of thermodynamics, whereas resistance may increase or decrease depending on the degradation mechanism. Commercial resistors were tested in order to characterize the damage induced by power surges. Resistors were biased with constant and pulsed voltage signals, leading to power dissipation in the range of $4-8 \mathrm{~W}$, which is well above the $0.25 \mathrm{~W}$ nominal power to initiate failure. Entropy was inferred from the added power and temperature evolution. A model is proposed to understand the relationship among resistance, entropy, and damage. The power surge dissipates into heat (Joule effect) and damages the resistor. The results show a correlation between entropy generation rate and resistor failure. We conclude that damage can be conveniently assessed from irreversible entropy generation. Our results for resistors can be easily extrapolated to other systems or machines that can be modeled based on their resistance. (C) 2015 AIP Publishing LLC.
\end{abstract}

[http://dx.doi.org/10.1063/1.4934740]

\section{INTRODUCTION}

Electrical circuit performance relies on the quality of their components. These components may become damaged or wear over time, jeopardizing circuit performance. A good understanding of their reliability is necessary to predict system lifetime. Determining reliability has been approached from various perspectives: electrical modeling, statistical distributions, and thermodynamic evolution. Electrical modeling characterizes the electrical parameters as abnormalities in impedance, current, or power consumption. Statistical approaches for modeling damage evolution include curve fitting approaches, such as Weibull distributions and Kalman filters. ${ }^{1,2}$ This knowledge is based on many a priori measurements and not directly connected to a direct wear out property. A potentially interesting field that has attracted less attention is thermodynamics. ${ }^{3} \mathrm{~A}$ branch of thermodynamics studies systems that undergo irreversible processes, including classical irreversible thermodynamics (CIT). From a theoretical perspective, entropy generation rate $\sigma_{\mathrm{s}}$ is a parameter that "is important in engineering because the product $T \sigma_{\mathrm{s}}$ is a measure of the degradation or dissipation of energy in engines, and its minimization may be useful to enhance their efficiency." 4 This approach has been applied to the study of mechanical damage and wear in solid materials. ${ }^{5-8}$ Khonsari and Amiri have carried out a comprehensive investigation of thermodynamics of mechanical failure. ${ }^{9}$ Also, Naderi et al. have investigated heat generation during mechanical stress in beams and proposed an entropy threshold for damage assessment. ${ }^{8,10}$

Entropy is only sparsely applied in electrical applications. However, the entropy generated from conductive

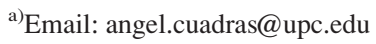

wires is well known in thermodynamics. ${ }^{4,11}$ The Joule effect is also an evident phenomenon. The electrical behavior following mechanical fatigue has also been evaluated, where mechanical damage has been related to electrical damage. ${ }^{5}$ In these mechanical systems, damage is monitored using the parameter $D,{ }^{12}$ where $0 \leq \mathrm{D} \leq 1, D=0$ means no damage, and $D=1$ means total damage. Basaran and $\mathrm{Yan}^{6}$ have pioneered the introduction of entropy as a damage metric in electromigration characterization, i.e., lattice degradation due to electron scattering. Thus, the combination of current and voltage as electrical variables, along with entropy from thermodynamics, could lead to an accurate description of electrical damage. ${ }^{6,13,14}$ Entropy has also been introduced for understanding the physics leading to the failure of oxides in order to obtain accurate models of their reliability. ${ }^{15,16}$ Reversible entropy is widely used in adiabatic computing, ${ }^{17}$ where heat dissipation must be minimized, and in electrochemical battery research, for characterizing their charge state and health. ${ }^{18,19}$

Recently, Amiri and Modarres ${ }^{20}$ have reviewed the possibilities for successfully implementing entropy as a parameter for measurement and instrumentation. While mainly theoretical, they highlighted its viability in electrical and mechanical systems. Nonetheless, entropy has not yet reached maturity for system monitoring. In this study, we investigate entropy evolution to characterize commercial resistor damage, with the aim of generalizing the effects of resistive thermal dissipation for any dissipative resistance-modeling system. Our objective is to demonstrate that entropy is a valuable parameter for studying resistor deterioration, even more so than resistance. Resistance can increase or decrease due to several effects, including electronic dispersion, mechanical strain (Bridgman law), temperature, electromigration, oxidation, and corrosion. Resistor failures can be due to 
overheating or overvoltage, which may lead to either open or short circuits. $^{15}$

We attempt to combine the thermodynamic framework proposed by the Basaran group ${ }^{2,6,14,21}$ and the parametric approach of damage and failure reliability as investigated by the Feinberg group. ${ }^{3,22,23}$ The final aim is to explain both short and open circuit resistor behavior with a single model based on positive irreversible entropy generation. Thus, a threshold for maximum allowable damage can be established, which has been suggested in mechanical damage studies by Naderi. ${ }^{8}$ This approach could pave the way for successfully implementing entropy as a parameter for assessing the deterioration in other electrical systems with parasitic resistance, including capacitors, inductors, power lines, and batteries.

\section{THEORETICAL APPROACH}

Entropy is a thermodynamic function of intrinsic and extensive states, and commonly used to describe irreversible processes. Generally, a system satisfies the law of entropy balance ${ }^{4}$ when

$$
\dot{S}=\dot{S}_{\mathrm{e}}+\dot{S}_{\mathrm{i}}
$$

where the subindices $e$ and $i$ refer to external (entropy exchange) and internal entropy (entropy generation), respectively. The dot over the variable represents a time derivative, i.e., $\dot{S}=d S / d t$. We impose the approximation of local equilibrium, i.e., the system is locally stable and the local and instantaneous relationships between thermodynamic quantities in a system out of equilibrium are the same as those for a uniform system in equilibrium. In this regard, entropy remains a valuable state function, even under non-equilibrium conditions. In accordance with the second law of thermodynamics, $\dot{S}_{\mathrm{i}} \geq 0$. Equation (1) can be rewritten in differential form using the volume integral of $\sigma_{\mathrm{s}}$, the rate of entropy production,

$$
\dot{S}_{\mathrm{i}}=\int_{V} \sigma_{\mathrm{s}} d V
$$

and the entropy flux, $J_{\mathrm{s}}$, integrated through the surface, $\Sigma$, using the normal projection $n$,

$$
\dot{S}_{\mathrm{e}}=-\int_{\Sigma} J_{\mathrm{s}} \cdot n d \Sigma .
$$

Hence,

$$
\rho \frac{\partial s}{\partial t}=-\nabla J_{\mathrm{s}}+\sigma_{\mathrm{s}},
$$

where $\rho$ is the mass density $(S=\rho s)$ and $s$ is the specific entropy, and thus, $\sigma_{s} \geq 0$ is satisfied. ${ }^{4}$ With regards to electrical conductors, electrical current dissipates heat energy (Joule effect) so that the generated entropy is related to the added power, such that

$$
T \rho \sigma_{\mathrm{s}}=R I^{2}
$$

where $R$ is the element resistance, $I$ the electrical current, and $T$ is the resistor temperature. This expression is analogous to the Gouy-Stodola theorem, ${ }^{24}$ which states that whenever a system operates irreversibly, it destroys work at a rate that is proportional to the system's rate of entropy generation. The theorem is usually applied to thermal machines exchanging mechanical work with an external atmosphere. If we related this to a resistor, as illustrated in Fig. 1, the added electrical power, $P$, in the resistor, due to the current flowing to it, $I$, and the voltage drop, $V$, generate entropy equal to

$$
\dot{S}=\frac{P}{T}=\frac{I V}{T} .
$$

The power dissipated in a resistor through the Joule effect is typically assumed to dissipate as heat to the environment, which is normal for resistor operation. However, it is also true that a continually biased resistor deteriorates. We propose to study entropy dissipation using Eq. (1), where the usual heat flux entropy is $\dot{S}_{\mathrm{e}}$ and the resistor degradation is assessed from $\dot{S}_{\mathrm{i}}$. This would demonstrate a dependence of friction dissipation in sliding solids through three processes: rise in temperature; wear particle generation; and the entropy changes associated with material transformation in the interface. ${ }^{7,25}$ In this work, we determine a correlation between damage entropy and the resistor's resistance evolution, which may help for resistor damage diagnosis and prognosis. The form of $\dot{S}_{\mathrm{i}}$ will be discussed later in light of the experimental results. Finally, entropy change is obtained by integrating the entropy rate:

$$
\Delta S=\oint \dot{S} d t .
$$

\section{MATERIALS AND METHODS}

Commercially available $0.25 \mathrm{~W}$ carbon film resistors were investigated. These resistors are made of carbon film deposited onto a ceramic rod and covered by an electrically insulating epoxy resin, which is also a good thermal conductor. Their main characteristics are described in Table I. The resistors used for testing ( $\left.R_{\text {DUT }}\right)$ were placed in a voltage divider, with $R_{\mathrm{D}}$ in series to monitor current $\left(R_{\mathrm{D}}<R_{\mathrm{DUT}}\right.$, usually $R_{\mathrm{D}}=0.1 R_{\mathrm{DUT}}$ ), as depicted in Figs. 1 and 2 . Resistors were biased in order to measure their power dissipation. Temperature was monitored using a PT1000 sensor that was

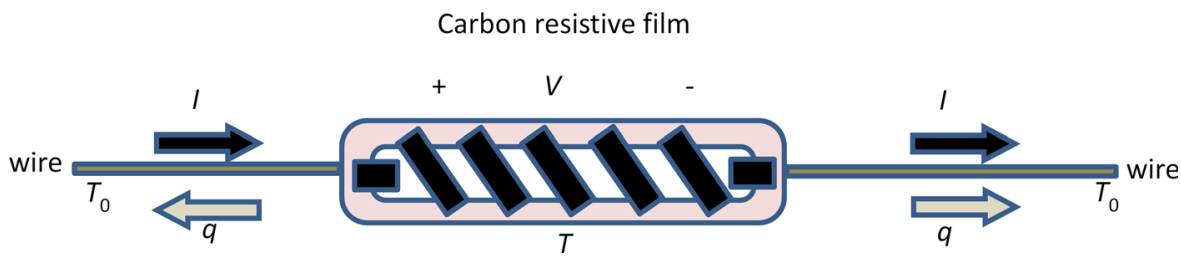

FIG. 1. Resistor under voltage bias $V$, current flow $I$, and emitting heat $q . T_{\mathrm{o}}$ is the environmental temperature and $T$ is the actual resistor temperature. 
TABLE I. Resistor data according to the datasheet.

\begin{tabular}{lc}
\hline \hline Property & Values \\
\hline Tolerance & $5 \%$ \\
Rated temperature & $70^{\circ} \mathrm{C}$ \\
Operating temperature range & $-55-155^{\circ} \mathrm{C}$ \\
Maximum voltage & $250 \mathrm{~V}$ \\
Rating wattage at $70^{\circ} \mathrm{C}$ & $0.25 \mathrm{~W}$ \\
Load life & $\pm 2 \% \Delta \mathrm{R} / \mathrm{R}$ for 1000 operating hours at rated continuous working voltage (RCWV) with duty cycle of $1.5 \mathrm{~h}$ on and \\
& $0.5 \mathrm{~h}$ off at $70^{\circ} \mathrm{C} \pm 2{ }^{\circ} \mathrm{C}$ ambient \\
Short time overload & Resistance change rate is $\pm(1 \%+0.05 \Omega)$ with no mechanical damage. Applied voltage 2.5 times the RCWV for $5 \mathrm{~s}$ \\
Temperature cycling & Resistance change rate is $\pm(1 \%+0.05 \Omega)$ with no mechanical damage in the operating temperature range \\
\hline \hline
\end{tabular}

mechanically attached to the resistor and thermally connected with a heat sink compound (Dow Corning 340).

Voltage dividers were biased with voltages ranging from $7 \mathrm{~V}$ to $10 \mathrm{~V}$ using a voltage source (Promax FAC662B). Both continuous and pulsed biases, with a duty cycle of $66 \%$, were investigated $\left(30 \mathrm{~s} / 15 \mathrm{~s}\right.$ On/OFF). $R_{\text {DUT }}$ was also investigated when placed in air at room temperature and covered using a thermally insulating polymer inside of a metallic box (thermal conductivity is approximately $0.06 \mathrm{~W} \mathrm{~m}^{-1}$ $\mathrm{K}^{-1}$ ) to minimize heat exchange with the surroundings. Current, voltage, and temperature were acquired using a microcontroller-based data acquisition system. Data were transferred to a computer and manipulated using Eqs. (5) and (6) to infer entropy accumulation and entropy rate generation. From these data, knowledge of the circuit's deterioration was obtained instantaneously.

\section{RESULTS}

Here, we summarize the results obtained from electrically stressing $10 \Omega$ resistors under various experimental conditions, in order to determine the relationship between resistor damage and entropy generation.

\section{A. Measured parameters under different conditions}

Resistors were biased at a constant voltage. The added power, voltage, current, temperature, resistance, and $\dot{S}$ were monitored for several resistors in air and covered, under continuous and pulsed modes. We observe that the power slightly increases with a decrease in resistance and an increase in temperature. $\dot{S}$ exhibits a change in its behavior at the same time as resistance, as illustrated in Figs. 3 and 4, for continuous and pulsed biases.

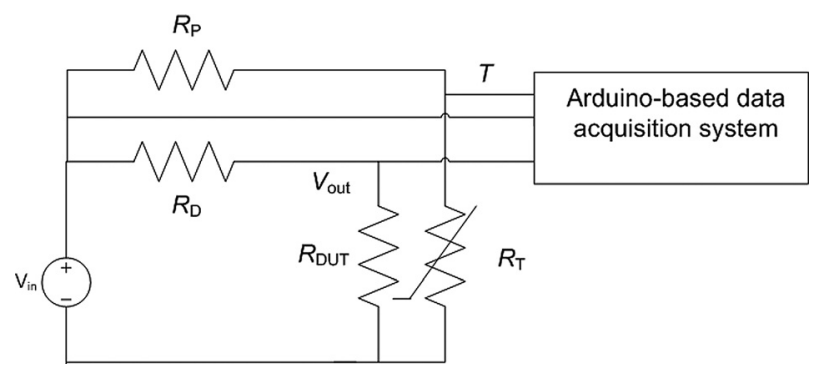

FIG. 2. Experimental circuit setup. $R_{\text {DUT }}$ belongs to the electrical voltage divider and $R_{\mathrm{T}}$ belongs to the PT1000 sensor circuit.

\section{B. Relationship between magnitudes}

Resistance and entropy are found to be directly related, as illustrated in Fig. 5. Resistance decreases with time until failure. We compute $\dot{S}$ according to Eq. (6), and find that $\dot{S}$ evolves in a manner similar to the evolution of resistance. This correlation is found in all of the studied cases. Thus, $\dot{S}$ may be a valuable indicator of resistor performance.

Figure 6 illustrates that the time to failure decreases when the dissipated power in the resistor increases, as expected. Also, under identical electrical conditions, the stressed resistor fails earlier when covered than in air, which is also expected (see Fig. 7). Moreover, the resistance evolves differently when the resistor is biased larger or smaller. In the first case, the resistance decreases until failure. In the second case, the resistance increases following a common logarithmic rule for a fatigue aging process, ${ }^{3}$ and



FIG. 3. Graph comparison among injected power, temperature, $\dot{S}$, and resistance as a function of time for continuous injection at $9.5 \mathrm{~V}$. The vertical line flags the failure time, indicating that $\dot{S}$ is able to predict the failure. The line connecting the points is to guide the eye. 


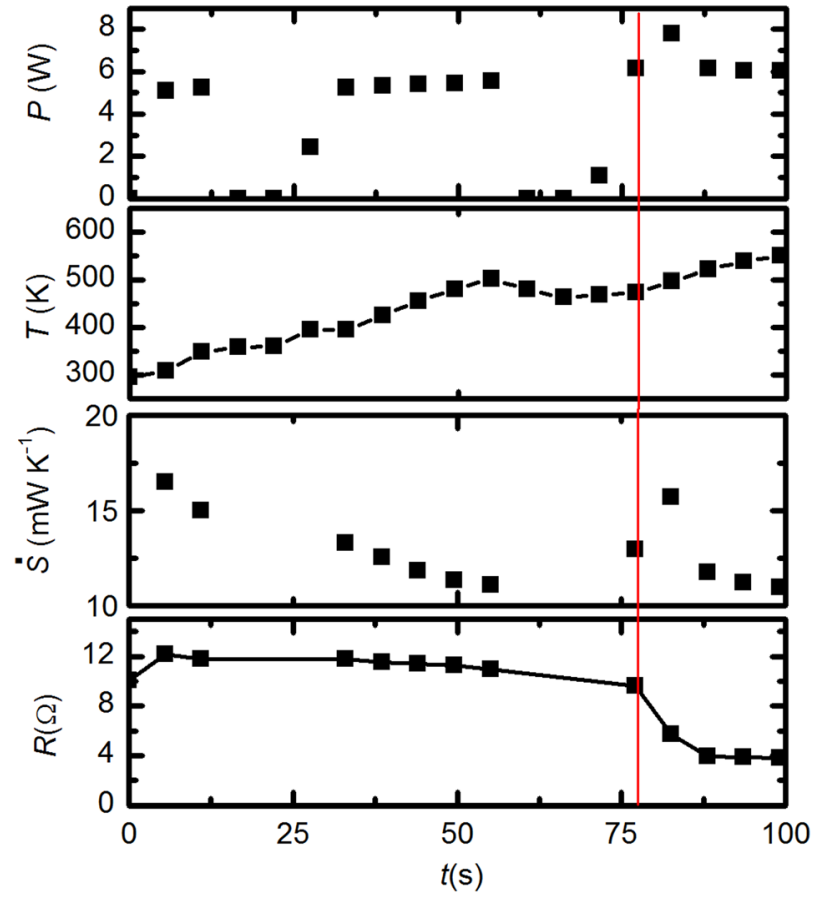

FIG. 4. Graph comparison among injected power, temperature, $\dot{S}$, and resistance as a function of time. The vertical line highlights the failure time and demonstrates that $\dot{S}$ can be an indicator of resistor failure. The line connecting the points is to guide the eye. Singular points where injected power is zero (disconnection) have been removed for clarity.

does not break down (see Fig. 8). Also, the time scales of both effects are very different, as demonstrated by a comparison of Figs. 7 and 8.

Now that the electrical behavior has been discussed, we discuss the thermal behavior. $\dot{S}$ exhibits a trend that is the same as that followed by electrical measurements. In Fig. 9, we plot $\dot{S}$ for various continuous biases in air. $\dot{S}$ decreases as the bias decreases. Finally, we integrate $\dot{S}$ to find the total entropy variation, $\Delta S$, as depicted in Fig. 10. We find that entropy increases faster in cases where failure occurs earlier. However, the total entropy generation is not a useful parameter to assess resistor performance, because it is due to both thermal flux dissipation and system degradation. Specifically, resistors biased at lower voltages have a larger entropy



FIG. 5. Comparison between resistance evolution and $\dot{S}$ in the resistor at $8 \mathrm{~V}$ in air and under continuous excitation.

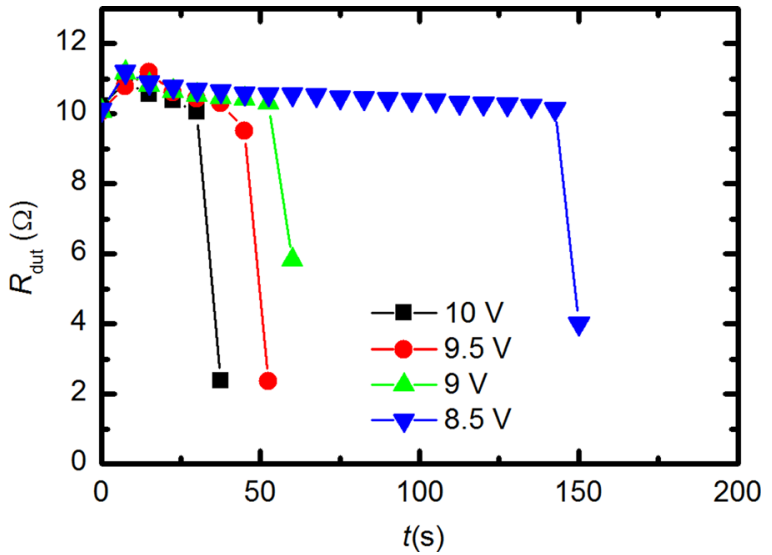

FIG. 6. Resistor failure due to various biases in $10 \Omega$ in air.

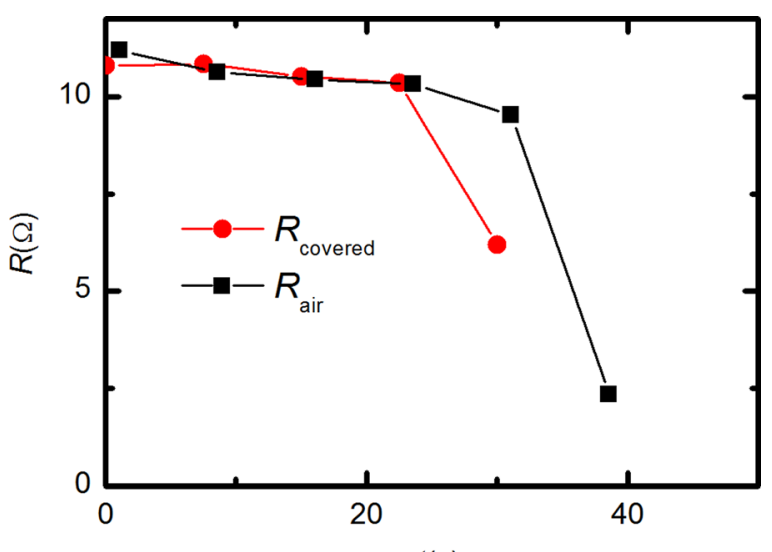

$t(\mathbf{s})$

FIG. 7. $R$ variation for a $10 \Omega$ resistor in a closed and open environment applying an input voltage of $9.5 \mathrm{~V}$.

variation because they exchange more entropy with the surroundings as heat flux.

\section{Reliability projections}

Here, we conduct a reliability projection for these components to summarize our findings. We plot the time-to-failure, defined as the time when the resistance increases above $10 \%$ over the nominal value due to fatigue or when the resistor

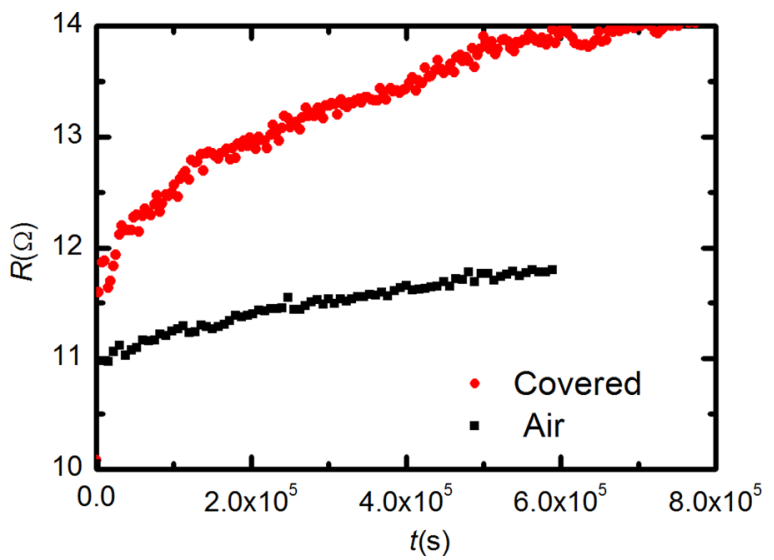

FIG. 8. Evolution of resistance at $7 \mathrm{~V}$ in air and covered, under continuous excitation. Notice the time span in comparison to Fig. 7. 


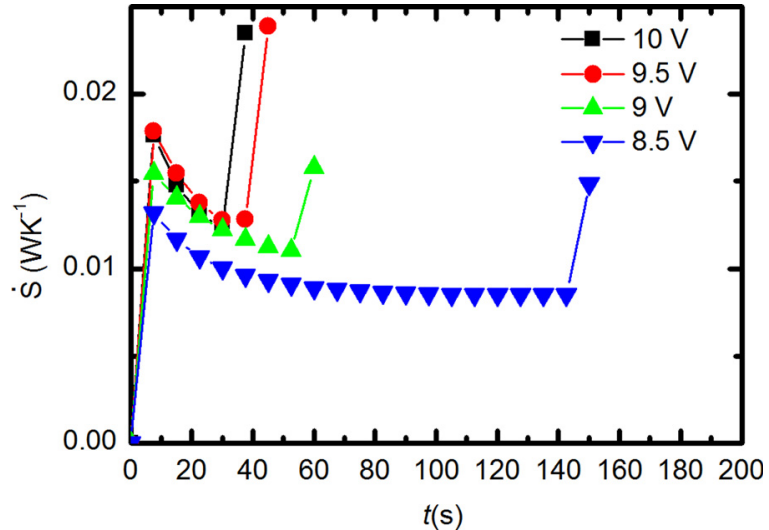

FIG. 9. Evolution of $\dot{S}$ in resistors biased using a constant voltage in air.

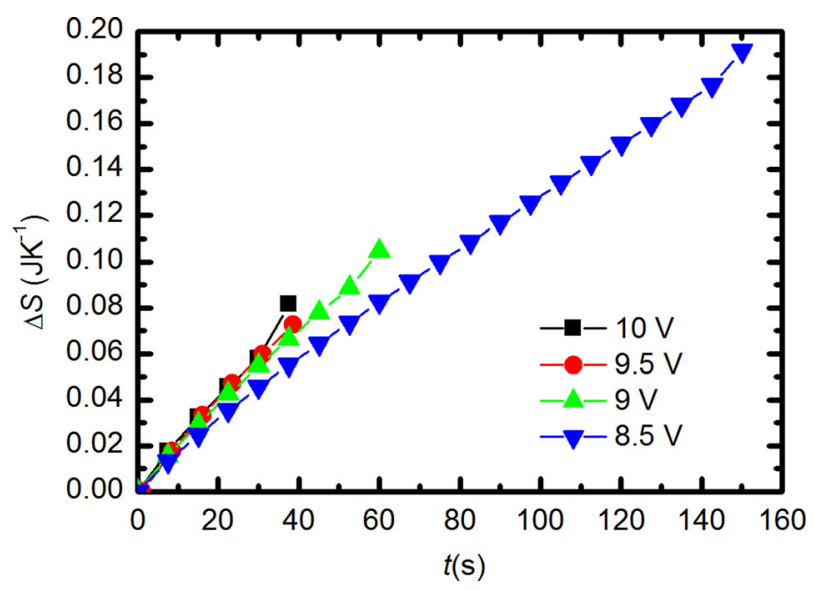

FIG. 10. Total entropy generation in the resistor.

fails, for the injected power, as depicted in Fig. 11. This time-to-failure is the same for both resistance failure and $\dot{S}$. Therefore, if the entropy of the system is measured, the system's performance can be projected. This would apply to any system that dissipates power and works under heated conditions: electrical, mechanical, or even chemical. However, we will see that this representation is not sufficient to describe resistance failure because it takes into

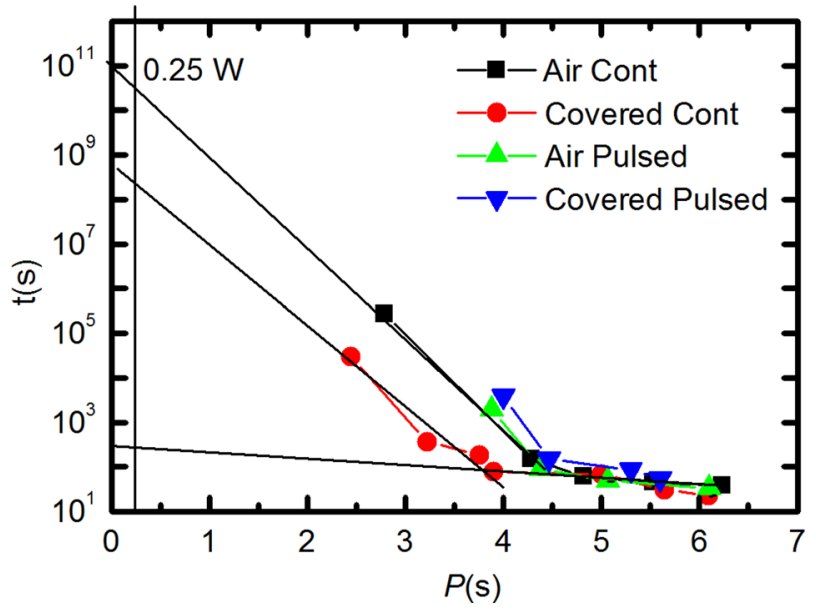

FIG. 11. Lifetime projection estimation as a function of injected power $P$. account injected power rather than degradation energy, as discussed for Fig. 13.

\section{DISCUSSION}

First, we consider the well-known resistor-reversible temperature dependence. Second, we discuss the entropy generation rate and how to distinguish the thermal generation and aging effects. Third, we consider the physical mechanism that degrades the resistor. Finally, we discuss the entropy generation rate as a valuable diagnosis parameter.

With respect to reversible temperature dependence, models based on free electron gas models provide a wellknown expression relating temperature to resistance,

$$
R=R_{\text {nom }}\left(1+\alpha\left(T-T_{r e f}\right)\right),
$$

where $\alpha= \pm 350 \mathrm{ppm}{ }^{\circ} \mathrm{C}^{-1}$. For the tested $10 \Omega$ nominal resistors, this leads to an increase of approximately $1 \Omega$, depending on the resistor, as shown in Figs. 6 and 7. This change in resistance is reversible and does not damage the device.

\section{A. Damage characterization}

We now focus on the damage characterization. According to Lemaitre, ${ }^{5}$ resistor damage is given by a phenomenological relationship,

$$
I=\frac{I_{0}}{1-D},
$$

where $D$ is damage, $I$ is the actual current, $I_{0}$ is the nominal current in a fresh resistor, and $0 \leq D \leq 1$, where $D$ refers to the decrease in elasticity modulus. ${ }^{26}$ The use of a damage parameter, $D$, has also been inferred from statistical principles. ${ }^{21,27}$ However, this relationship is not considered useful to explain our results because $I$ can either increase or decrease, which is not explained with this equation. In electrical degradation, two possible mechanisms are found: cascade conduction and resistance increase. In either case, current increases or decreases. Since damage can be studied as an irreversible process, $\sigma_{\mathrm{s}} \geq 0$. So after recovering Eq. (1), we can rewrite it as

$$
\begin{gathered}
\dot{S}=\dot{S}_{\mathrm{e}}+\dot{S}_{\mathrm{i}}, \\
\frac{V I}{T}=\frac{\dot{Q}_{\mathrm{e}}}{T}+\dot{S}_{\mathrm{i}}, \\
V I=\dot{Q}_{\mathrm{e}}+\dot{S}_{\mathrm{i}} T,
\end{gathered}
$$

where $\dot{Q}_{\mathrm{e}}$ is the exchanged heat with the surroundings. Since $V, I$, and $T$ are known, we can plot $V \cdot I$ as a function of $T$, as depicted in Fig. 12. A similar approach was used for determining degradation in sliding contacts. ${ }^{28}$ The independent term is related to entropy flow, whereas the slope is related to damage entropy generation. The critical point is to determine the behavior of $\dot{S}_{\mathrm{i}}$. According to CIT, the behavior should be expressed as the product of force and flux. If one considers that the material's structure changes, a change in 


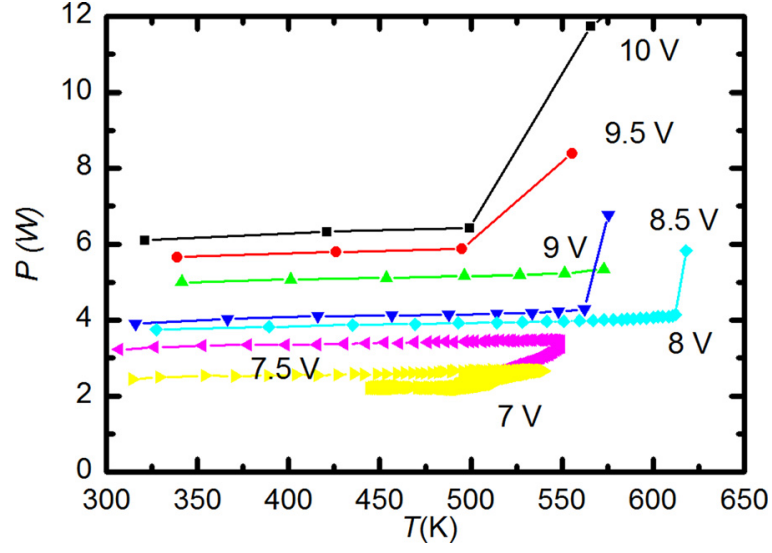

FIG. 12. Power added to the resistor $P$ plotted against temperature for covered and continuous data. The slope slightly increases, whereas the heat flux intercept decreases.

stress and strain in the material can be expected. ${ }^{12,27,29}$ The intrinsic or mechanical dissipation, $\phi_{\text {int }}$, is written as ${ }^{5,26}$

$$
\phi_{\mathrm{int}}=\sigma: \dot{\varepsilon}^{p}-A_{\mathrm{k}} \cdot \dot{V}_{\mathrm{k}}
$$

where $\sigma$ and $\dot{\varepsilon}^{\mathrm{p}}$ are the stress and strain tensors, respectively, and $A_{\mathrm{k}}$ and $\dot{V}_{\mathrm{k}}$ stand for other internal variables able to describe material changes. The first term is dissipation due to plastic deformation, induced by the product of the strain tensor and applied stress, and the second term is related to the nonrecoverable energy stored in the material, i.e., dilatation dissipation. This second term accounts for $5 \%-10 \%$ of the total dissipation, so it is usually negligible. ${ }^{5,21}$ Stress would be due to the generation of defects, including interstitials and dislocations, governed by electrical potentials. One of the critical issues of this work is to justify fatigue (resistance increase) and failure (resistance increase) as irreversible processes with positive entropy generation. Kluitenberg ${ }^{29}$ developed an expression that relates the entropy generation rate to material strains (see Eqs. (2.6) and (5.1) in Ref. 29, and consider only the mechanical terms),

$$
\begin{aligned}
\sigma_{\mathrm{si}} & =\frac{1}{T}\left(-\sum_{\alpha, \beta=1}^{3} P_{\alpha, \beta}^{(v)} \frac{\mathrm{d} \tilde{\varepsilon}_{\alpha \beta}}{\mathrm{d} t}+\sum_{\alpha, \beta=1}^{3} \tau_{\alpha \beta}^{i} \frac{\mathrm{d} \varepsilon_{\alpha \beta}^{i}}{\mathrm{~d} t}-3 P^{(v)} \frac{\mathrm{d} \varepsilon}{\mathrm{d} t}+3 \tau^{i} \frac{\mathrm{d} \varepsilon^{i}}{\mathrm{~d} t}\right), \\
& =\frac{1}{T}\left(\eta^{(3)} \sum_{\alpha, \beta=1}^{3}\left(\frac{\mathrm{d} \tilde{\varepsilon}_{\alpha \beta}}{\mathrm{d} t}\right)^{2}+3 \eta^{(4)}\left(\frac{\mathrm{d} \varepsilon}{\mathrm{d} t}\right)^{2}+\eta^{(1)} \sum_{\alpha, \beta=1}^{3}\left(\tau_{\alpha \beta}^{i}\right)^{2}+3 \eta^{(2)}\left(\tau^{i}\right)^{2}\right),
\end{aligned}
$$

where $P_{\alpha \beta}^{(v)}$ is the viscous pressure tensor, $\tilde{\varepsilon}_{\alpha \beta}$ and $\varepsilon_{\alpha \beta}^{i}$ are the total and inelastic strain tensors, and $\tau_{\alpha \beta}^{i}$ and $\tau^{i}$ are the stress tensors. Both elastic and inelastic strains contribute to entropy generation. The second term is a linearized approximation described by the constants $\eta^{(1)}, \eta^{(2)}, \eta^{(3)}$, and $\eta^{(4)}$. The key point for our approach is that this expression is temperature independent. The entropy generation rate is definitely positive because all of the strain terms are quadratic, i.e., regardless of strain generation (fatigue or failure), entropy will be positive, as expected from the second law of thermodynamics. In our opinion, this was not evident in previous studies based on $D$ parameters. ${ }^{5,6,13,27}$ Thus, we plot the curve of added power to the resistor as a function of temperature, $P(\mathrm{~T})$, as illustrated in Fig. 12, which shows linear behavior, and we can estimate $\dot{S}_{\text {i }}$ from the linear fit.

Alternatively, the term related to entropy flow, the thermal entropy generation rate per unit volume, is

$$
\sigma_{\mathrm{se}}=\rho \frac{d s}{d t}+\nabla J_{\mathrm{s}}=q \nabla\left(\frac{1}{T}\right)=\frac{q^{2}}{\lambda T^{2}},
$$

where $\lambda$ is thermal conductivity. ${ }^{4}$ This expression can be further developed if heat flux $q\left(\mathrm{Wm}^{-2}\right)$ is characterized. Thermal conduction takes place through surface contact with the environment and through the resistor cable connections, and described by the Fourier conduction law as ${ }^{4}$

$$
q=-\lambda \nabla T
$$

Generation rate is related to entropy generation according to ${ }^{11}$

$$
\sigma_{\mathrm{se}}=\frac{1}{\lambda} \frac{(\nabla T)^{2}}{T^{2}} .
$$

We do not have access to thermal gradients, but we can quantify the total heat transferred, $\dot{Q}_{\mathrm{e}}$.

We found a linear trend in the $P-T$ representation as illustrated in Fig. 12. Using Eq. (10), we fit linear regressions to experimental data, as plotted in Fig. 13, and summarize



FIG. 13. Lifetime projection estimation as a function of $\dot{S}_{\mathrm{i}}$ for shortcircuited resistor failure. 
TABLE II. Linear regression fittings for entropy generation rate in covered and air measurements. M1 and M2 distinguish between short and open failure mechanisms.

\begin{tabular}{|c|c|c|c|c|c|}
\hline \multirow[b]{2}{*}{$\begin{array}{l}\text { Bias } \\
\text { voltage }(\mathrm{V})\end{array}$} & \multirow[b]{2}{*}{$\begin{array}{c}\text { Input } \\
\text { power }(\mathrm{W})\end{array}$} & \multicolumn{2}{|c|}{ Covered continuous } & \multicolumn{2}{|c|}{ Air continuous } \\
\hline & & $\begin{array}{c}\text { Slope } \\
\left(\mathrm{mW} \mathrm{K}^{-1}\right)\end{array}$ & $\begin{array}{l}\text { Intercept } \\
\text { (W) }\end{array}$ & $\begin{array}{c}\text { Slope } \\
\left(\mathrm{mW} \mathrm{K}^{-1}\right)\end{array}$ & $\begin{array}{l}\text { Intercept } \\
\text { (W) }\end{array}$ \\
\hline 10 & 6 & 1.8 & 5.5 & 1.87 & 5.57 \\
\hline 9.5 & 5.6 & 1.4 & 5.16 & 2.53 & 4.77 \\
\hline 9 & 5 & 1.2 & 4.57 & 2.08 & 4.20 \\
\hline 8.5 & 4.1 & 1.3 & 3.53 & 1.55 & 3.77 \\
\hline 8 & 3.7 & 1.1 & 3.38 & & \\
\hline 7.5 M1: & 3.2 & 0.95 & 2.96 & & \\
\hline 7.5 M2: & & 25 & 10.5 & & \\
\hline $7 \mathrm{M1}$ : & 2.4 & 0.79 & 2.22 & & \\
\hline 7 M2: & & 12.6 & 4.03 & & \\
\hline
\end{tabular}

the slope and intercept terms in Table II. The slope is directly related to $\dot{S}_{\mathrm{i}}$, and we observe that the slope and thus $\dot{S}_{\mathrm{i}}$ become larger as the resistor degradation rate increases, due to the surge in power. The intercept is related to heat flux. When the dissipated power increases, the heat flux also increases, as expected. Both covered and exposed experimental curves exhibit linear behavior, but their slopes are different (see Table II). Based on these findings, it is reasonable to assume that their aging mechanisms are the same. ${ }^{10}$

For low power surges, $\dot{S}_{\mathrm{i}}$ exhibits different behavior, i.e., two slopes, M1 and M2, are possible. Consequently, distinguishing between the two degradation mechanisms is possible with this method. $\dot{S}_{\mathrm{i}}$ also behaves linearly, as expected from the irreversible aging due to material stress. In turn, $\dot{S}_{\mathrm{i}}$ can be characterized by identifying the constants in Eq. (12), which depend on the material. This finding enables one to understand material generation and energy defects using entropy monitoring. Although we did not aim to investigate the behavior of resistor materials in this work, it might be the basis of a future investigation.

We find that the energy needed for aging is $T \cdot \dot{S}_{\mathrm{i}}$. The product of the slope and maximum temperature produces values between $0.5 \mathrm{~W}$ and $0.9 \mathrm{~W}$. The input power ranges from $2.4 \mathrm{~W}$ to $6.2 \mathrm{~W}$. Thus, in this case, the power devoted to resistor degradation is roughly $7 \%-9 \%$ of the input magnitude, signifying that the resistor dissipates heat and degrades, before it finally fails. We emphasize that this percentage is large because the resistors are highly stressed, above their nominal values, which damages the resistors during continuous use. This representation is more accurate for resistor lifetime predictions than that depicted in Fig. 11, because it only considers the effects of degradation, whereas the former includes entropy exchange. This approach can be useful for resistive systems that are not specially designed as resistors, which deteriorate when electrical current flows through them. Resistors have been specially designed for this purpose. Thus, once we have demonstrated this behavior in the general case, it can be applied to other particular cases.

We conclude with a discussion of the contributions of both elastic and inelastic strain, in accordance with Eq. (12). While inelastic strain clearly results in irreversible material

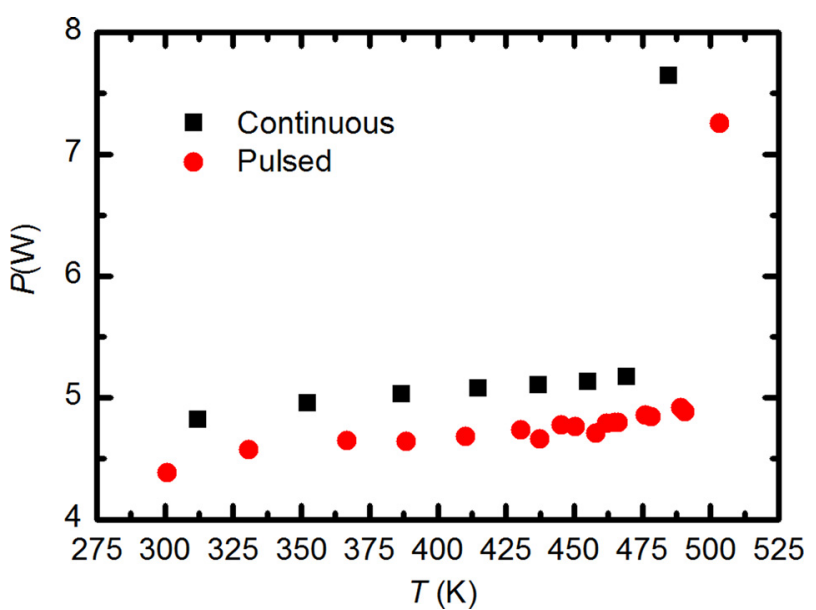

FIG. 14. Comparison between continuous and pulsed entropy generation for resistors biased at $9 \mathrm{~V}$ in air.

damage, elastic strain can decrease. Thus, if we bias a resistor at large voltage, we find that its resistance decreases (see Fig. 6), but when it cools down, its resistance increases, up to $14.7 \Omega$ and $11.9 \Omega$ for $9 \mathrm{~V}$ and $8 \mathrm{~V}$ biases, respectively. After the resistors have failed, analyses of the resistors might be necessary for discriminating the failure mechanism. To further verify the elastic stress influence, we compared the results of $T \cdot \dot{S}_{\mathrm{i}}$ for continuous and pulsed signals, as shown in Fig. 14. Similar behavior is found in both cases. Slopes are $2.08 \pm 0.16 \mathrm{~mW} \mathrm{~K}^{-1}$ and $1.95 \pm 0.23 \mathrm{~mW} \mathrm{~K}^{-1}$, whereas intercepts are $4.20 \pm 0.07 \mathrm{~W}$ and $3.90 \pm 0.1 \mathrm{~W}$, respectively. The intercept is smaller in the pulsed case because the average power is smaller due to the duty cycle. However, the degradation mechanisms seem consistent in both cases, confirming the hypothesis that temperature is independent of $\dot{S}_{\mathrm{i}}$, as proposed in Eq. (12). Also, from this expression, we infer that since the slope is much similar in both cases, the small differences can be attributed to elastic stresses in the material.

\section{B. Degradation}

Depending on the degradation mechanism, resistor resistance can increase, because as described by percolation models, thermal dissipation generates activated defects that behave as open circuits ${ }^{30}$ or decreases if conductive paths are present in the material ${ }^{31}$ due to dielectric breakdown as electrons find a ballistic path. Dielectric breakdown occurs during heating, but when it cools down, the resistance increases and then degrades until it is reheated. Electromigration in the resistor is also possible and has been thermodynamically described in metals for current densities above $10^{4} \mathrm{~A} \mathrm{~cm}^{-2}$. For normal home utilities, current densities are approximately $10^{2} \mathrm{~A} \mathrm{~cm}^{-2}$ and do not result in aging or electromigration. ${ }^{27}$ In the case of our resistor experiments, current densities reach a maximum of about $500 \mathrm{~A} \mathrm{~cm}^{-2}$ (1 A in an estimated section of $2 \times 10^{-3} \mathrm{~cm}^{2}$ ), which would be low for a metal. However, in this case, we have a resistor, where electrical scattering is larger than that in metals. In both cases, entropy is positive as demonstrated in Refs. 27, 30, and 31. 
Electric Circuit

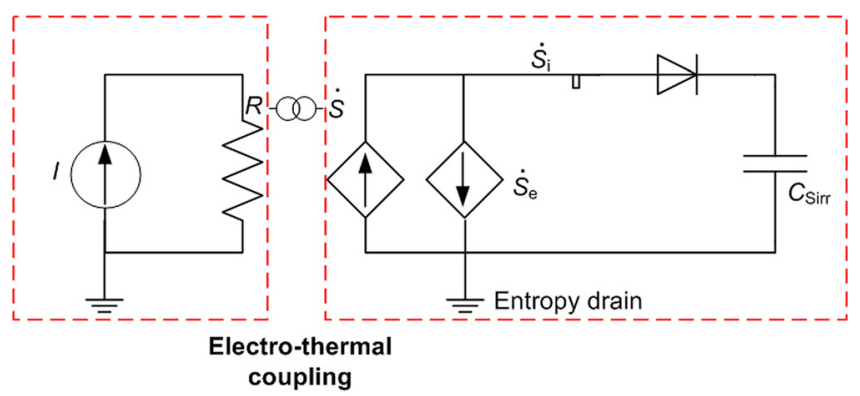

FIG. 15. Irreversible entropy damage model for a resistor generator. The electrical current $I$ flowing through $R$ dissipates energy at $R$. Irreversible entropy is generated $(\dot{S})$. Part of $\dot{S}$ is released to the environment as heat flux through $\dot{S}_{\mathrm{e}}$ and partially delivered for material degradation, $\dot{S}_{\mathrm{i}}$. We introduce a capacitor as a reservoir for cumulated entropy, $C_{\text {Sirr }}$, and a diode to reflect the irreversibility of the process.

\section{Entropy generation model}

From the experimental results, we have clearly determined that entropy varies in electrically stressed resistors, but some issues must be addressed. Note that, as expected from the second law of thermodynamics, intrinsic entropy always increases. Thus, it is convenient to evaluate the entropy delivered to the environment due to heat transfer, because the system is not adiabatic. In Sec. I, generated entropy was split between device entropy, $\Delta S_{\mathrm{i}}$, and the entropy transferred to the environment, $\Delta S_{\mathrm{e}}$. We propose an equivalent electrical circuit to describe this behavior, which is modeled using an element for each mechanism. The input power delivered to the resistor is described by a current source, I. This source is coupled to a dependent current source, $\dot{S}$, which describes the entropy generation rate in the resistor. The generated entropy is split into $\dot{S}_{\mathrm{e}}$ and $\dot{S}_{\mathrm{i}}$. This last term accounts for the irreversible processes in the resistor (fatigue and failure) and is modeled with a capacitor, $C_{\text {Sirr }}$, as a storage irreversible entropy reservoir, as illustrated in Fig. 15. When the capacitor reaches a certain threshold value, the system fails, which has been proposed in mechanical systems by Naderi. ${ }^{8}$

Accordingly, because internal entropy production leads to degradation of the system, ${ }^{2}$ it is possible to define a function,

$$
\dot{S}_{t f} \geq k_{t h} \dot{S}_{0}
$$

where $\dot{S}_{t f}$ and $\dot{S}_{0}$ are the failure and initial entropy generation rates, respectively. $k_{\text {th }}$ describes the constant threshold limit and can be tuned as a parameter for diagnoses and prognoses.

\section{CONCLUSIONS}

An entropy approach to commercial resistor reliability has been presented. In particular, the entropy generation rate has been demonstrated to be a good estimator for describing electrical circuit failure. The results presented here have demonstrated that entropy increase can be monitored in standard carbon commercial resistors under different biases and environmental conditions.
We have identified two different mechanisms leading to resistor failure. In both cases, a positive irreversible entropy generation rate was found. The entropy flow rate and generation rate inside the resistor have also been possible to identify, which clearly heralds deeper thermal characterization of electrical circuits. It is reasonable to assume that these results can be extended to other types of resistive elements, including electrochemically equivalent resistances, semiconductors, and digital logics.

Future studies must attempt to consider an entropy generation model for resistors to predict device lifetime as well as to validate the statistics of identical devices.

\section{ACKNOWLEDGMENTS}

This study was funded by the Spanish Government under Contract No. TEC2011-27397. V. J. Ovejas also acknowledges the financial support of a FPU contract given by MECD: Ministerio de Educación, Cultura y Deporte of the Spanish Government.

${ }^{1}$ E. A. Elsayed, Reliability Engineering (Wiley, 2006).

${ }^{2}$ C. Basaran, M. Lin, and H. Ye, Int. J. Solids Struct. 40, 7315 (2003).

${ }^{3}$ A. A. Feinberg and A. Widom, Reliab. IEEE Trans. 49, 136 (2000).

${ }^{4}$ G. Lebon, D. Jou, and J. Casas-Vázquez, Understanding Non-Equilibrium Thermodynamics: Foundations, Applications, Frontiers (Springer-Verlag, Germany, 2008).

${ }^{5}$ J. Lemaitre and J.-L. Chaboche, Mechanics of Solid Materials (Cambridge University Press, 1990).

${ }^{6}$ C. Basaran and C. Yan, J. Electron. Packag. 120, 379 (1998).

${ }^{7}$ M. Amiri and M. M. Khonsari, Entropy 12, 1021 (2010).

${ }^{8}$ M. Naderi, M. Amiri, and M. M. Khonsari, Proc. R. Soc. A Math. Phys. Eng. Sci. 466, 423 (2010).

${ }^{9}$ M. M. Khonsari and M. Amiri, Introduction to Thermodynamics of Mechanical Fatigue (CRC Press, 2012).

${ }^{10}$ M. Naderi and M. M. Khonsari, Mech. Mater. 46, 113 (2012).

${ }^{11}$ M. W. Zemansky and R. H. Dittman, Heat and Thermodynamics (McGraw-Hill, USA, 1990).

${ }^{12}$ G. A. Maugin, The Thermodynamics of Plasticity and Fracture (Cambridge University Press, Cambridge, UK, 1992).

${ }^{13}$ S. Li and C. Basaran, Mech. Mater. 41, 271 (2009).

${ }^{14}$ W. Yao and C. Basaran, J. Appl. Phys. 114, 103708 (2013).

${ }^{15}$ C. W. Lewis and J. J. Bohrer, in First Annual Symposium of Physics of Failure Electronics (1962), pp. 11-19.

${ }^{16}$ K. M. Browne, J. Non-Equilib. Thermodyn. 28, 147 (2003).

${ }^{17}$ E. Sanchez-Sinencio and A. G. Andreou, Low-Voltage/Low Power Integrated Circuits and Systems (Wiley Interscience, USA, 1998).

${ }^{18}$ Y. Reynier, J. Graetz, T. Swan-Wood, P. Rez, R. Yazami, and B. Fultz, Phys. Rev. B 70, 174304 (2004).

${ }^{19}$ R. Yazami, J. McMenamin, Y. Reynier, and B. T. Fultz, U.S. patent application PCT/US2010/026991 (16 September 2010).

${ }^{20} \mathrm{M}$. Amiri and M. Modarres, Entropy 16, 6434 (2014).

${ }^{21}$ C. Basaran and S. Nie, Int. J. Damage Mech. 13, 205 (2004).

${ }^{22}$ A. Feinberg, P. Ersland, V. Kaper, and A. Widom, in Annual Technical Meeting of the Institute of Environmental Sciences and Technology (Proc. Inst. Environ. Sci. Technol., 2000), pp. 231-236.

${ }^{23}$ A. Feinberg and A. Widom, IEEE Trans. Reliab. 45, 28 (1996).

${ }^{24} \mathrm{~A}$. Bejan, Advanced Engineering Thermodynamics (Wiley, New Jersey, USA, 2006).

${ }^{25}$ A. Ramalho and J. C. Miranda, Wear 260, 361 (2006).

${ }^{26} \mathrm{G}$. A. Maugin, The Thermomechanics of Plasticity and Fracture (Cambridge Texts in Applied Mathematics, Cambridge, 1992).

${ }^{27}$ J. A. Montemayor-Aldrete, C. Vázquez-Villanueva, P. Ugalde-Vélez, M. del Castillo-Mussot, G. J. Vázquez-Fonseca, A. Mendoza-Allende, and H. A. Coyotécatl, Phys. A 387, 6115 (2008).

${ }^{28}$ A. B. Aghdam and M. M. Khonsari, Wear 270, 781 (2011).

${ }^{29}$ G. A. Kluitenberg, Physica 35, 177 (1967).

${ }^{30}$ Z. Gingl, C. Pennetta, L. B. Kiss, and L. Reggiani, Semicond. Sci. Technol. 11, 1770 (1996).

${ }^{31}$ E. Miranda, D. Jimenez, and J. Suñe, Appl. Phys. Lett. 98, 253504 (2011). 